\title{
Welcome to the Third Workshop on Evolving Security and Privacy Requirements Engineering (ESPRE)
}

Welcome to the 3rd Evolving Security and Privacy Requirements Engineering (ESPRE) Workshop in Beijing, China on the $12^{\text {th }}$ of September 2016, co-located with the RE 2016 conference. The main focus of ESPRE is to bring together practitioners and researchers interested in security and privacy requirements. ESPRE probes the interfaces between requirements engineering and security \& privacy, and takes a step towards evolving security and privacy requirements engineering to meet a range of needs of stakeholders ranging from business analysts and security engineers to technology entrepreneurs and privacy advocates.

ESPRE's goal is to advance the scope of current research to consider novel approaches to the elicitation, analysis, and refinement of security and privacy requirements. In particular, ESPRE fosters new visions and novel applications of requirements engineering; leveraging expertise; establishing security standards; and fusing security design, implementation, and validation stages into requirements engineering. Interdisciplinary work is encouraged to investigate possibilities for aligning language and methodologies of other disciplines (e.g., legal analysis or health care procedures) with requirements engineering.

The workshop begins with a keynote address by Professor Lin Liu about security requirements engineering in the era of big data. The workshop continues with two more sessions. The "Security Requirements for Guidelines and Policies” session raises issues related to user-specific policies, deriving patterns from standards, and security requirements for policies for microservices. Our "Tools and Technology based Security and Privacy Requirements" session reveals design stories for argumentative security patterns, for code quality, and survey of privacy technologies. We are grateful to the programme committee for their hard work providing insightful and thoughtful feedback to all of the papers submitted. Each paper was assigned three (and often four) reviewers that judged the quality of the work and its ability to raise interesting debate during the workshop.

This is the third ESPRE workshop, a successor to the previous years' $1^{\text {st }}$ and $2^{\text {nd }}$ ESPRE workshops. The success of the first workshop of the series was shown in the 16 registrations, 2 keynotes and 8 paper presentations. The success of the second ESPRE workshop in 2015 resulted in 29 registrations, 2 keynotes, 5 paper presentations and 8 lightning talks. The ESPRE series of workshops builds on the success of prior workshops in security requirements engineering and secure software engineering, in particular the Security and Privacy Requirements Engineering (SPREE) Workshop in 2011, the International Workshop for Software Engineering for Secure Systems (SESS) series, and the Requirements for High Assurance Systems (RHAS) workshop series.

Kristian Beckers, Shamal Faily, Seok-Won Lee, and Nancy Mead

Organisers of the ESPRE Workshop 